\title{
Thermal annealing effects on the optical properties of high-indium InGaN epi-layers
}

\author{
Shih-Wei Feng ${ }^{1}$, Yung-Chen Cheng ${ }^{1}$, En-Chiang Lin ${ }^{1}$, Hsiang-Chen Wang ${ }^{1}$, C. C. Yang ${ }^{*}, 1$, \\ Kung-Jen $\mathrm{Ma}^{2}$, Ching-Hsing Shen ${ }^{3}$, L. C. Chen ${ }^{3}$, K. H. Kim ${ }^{4}$, J. Y. Lin ${ }^{4}$, and H. X. Jiang ${ }^{4}$ \\ ${ }^{1}$ Graduate Institute of Electro-Optical Engineering, Department of Electrical Engineering and Graduate \\ Institute of Electronics Engineering, National Taiwan University, 1, Roosevelt Road, Sec. 4, Taipei, \\ Taiwan \\ 2 Department of Mechanical Engineering, Chung Hua University, Hsinchu, Taiwan \\ ${ }^{3}$ Center for Condensed Matter Sciences, National Taiwan University, Taipei, Taiwan \\ ${ }^{4}$ Department of Physics, Kansas State University, Manhattan, Kansas 66506-2601, USA
}

Received 18 June 2003, accepted 26 July 2003

Published online 20 October 2003

PACS 78.55.Cr, 78.60.Hk, 78.66.Fd, 81.40.Ef

We compared the optical and material properties of two high-indium InGaN thin layers between the asgrown and post-growth thermally annealed conditions. In one of the samples, the major part of photoluminescence spectrum was shifted from the original yellow band into the blue range upon thermal annealing. Cathodo-luminescence (CL) spectra showed that the spectral shift occurred essentially in the photons emitted from a shallow layer of the InGaN film. The deeper layer in the as-grown film contributed blue emission because it had been thermally annealed during the growth of the shallow layer. The spectral shift was mainly attributed to the shrinkage of cluster size through spinodal decomposition upon thermal annealing. The attribution was supported by the observations in the CL, X-ray diffraction (XRD), timeresolved photoluminescence (TRPL) and, high-resolution transmission electron microscopy (HRTEM) results.

(C) 2003 WILEY-VCH Verlag GmbH \& Co. KGaA, Weinheim

1 Introduction and exprimental procedures Because the band gap of InGaN compound covers the whole visible wavelength range, it has the potential for color display and white-light lighting applications. Yellow and red luminescence from InGaN thin films of high indium contents have been reported [1]. However, optical characteristics and material microstructures of such a compound, particularly after thermal annealing, have not been well studied yet. Post-growth thermal annealing can usually changes the microstructures of InGaN compounds and hence their optical properties. In this paper, we report the thermal annealing effects on the optical and material characteristics of two high-indium InGaN layers with luminescence in the yellow and red ranges.

The sample used in this study was grown in a low-pressure metal-organic chemical vapor deposition reactor. A $\sim 1.2 \mu \mathrm{m} \mathrm{GaN}$ layer was deposited on a sapphire substrate with a $25 \mathrm{~nm} \mathrm{GaN}$ buffer layer. The GaN layer growth was followed by the deposition of an InGaN film with a thickness of $\sim 0.2 \mu \mathrm{m}$. The nominal indium contents were estimated slightly to be higher than $30 \%$ and $40 \%$, determined by energy dispersive $\mathrm{X}$-ray spectroscopy as well as the indium and gallium flow rates during crystal growth, in samples $\mathrm{Y}$ and R, respectively. Thermal annealing was conducted with $800^{\circ} \mathrm{C}$ for 30 minutes in ambient nitrogen. PL measurements were carried out with the $325 \mathrm{~nm}$ line of a $35 \mathrm{~mW} \mathrm{He}-\mathrm{Cd}$ laser for excitation. PLE experiments were conducted using quasi-monochromatic excitation light source from a xenon

* Corresponding author: e-mail: ccy@cc.ee.ntu.edu.tw, Phone: 886-2-23657624, Fax: 886-2-23652637 
lamp dispersed with a momochromator. The PLE detection photon energy was set at the PL peak energy. The TRPL measurements were performed using a Hamamatsu streak camera with time resolution about 5 ps. Frequency-doubled optical pulses with $100 \mathrm{fs}$ pulse width and $76 \mathrm{MHz}$ pulse repetition frequency were generated from a mode-locked Ti : sapphire laser for excitation. The excitation photon energies of TRPL were $3.1 \mathrm{eV}(400 \mathrm{~nm})$.

2 Optical and material characteristics Figure 1 shows the PL spectra at $10 \mathrm{~K}$ of the as-grown and annealed samples $\mathrm{Y}$ (upper portion) and R (lower portion). It is interesting to note that PL spectral peak for smaple $\mathrm{Y}$ has shifted from the yellow band (around $2.2 \mathrm{eV}$ ) to the blue band (around $2.75 \mathrm{eV}$ ) after thermal annealing. The blue emission contribution as a small side-lobe can also be observed in the $10 \mathrm{~K}$ PL spectrum of the as-grown sample. It is believed that PL emission essentially comes from a shallow layer of the sample. Its peak shifts due to microstructure alteration in the InGaN layer upon thermal annealing. This argument excludes the possibility that the yellow-band emission originated from defects in $\mathrm{GaN}[2]$. As for the annealed sample R, the luminescence shows multiple-band emission including red, green, and blue. With an appropriate annealing condition to adjust the weighting of different colors, we may obtain white light.

Figure 2 shows typical CL images of the as-grown (a) and annealed (b) samples Y, both with $15 \mathrm{kV}$ electrons. Light spots of a few $\mu \mathrm{m}$ in size confirm the cluster structures in the as-grown sample. The scanning electron microscopy image in the same region helps in identifying several large light spots in Fig. 2a as V-shape defects (those with dark cores at the centers) [3]. The dark cores correspond to the threading dislocations beneath the $\mathrm{V}$-shape defects. The emitted photons within those large light spots primarily come from the GaN layer (UV). The rest light spots of smaller sizes emit essentially yellow

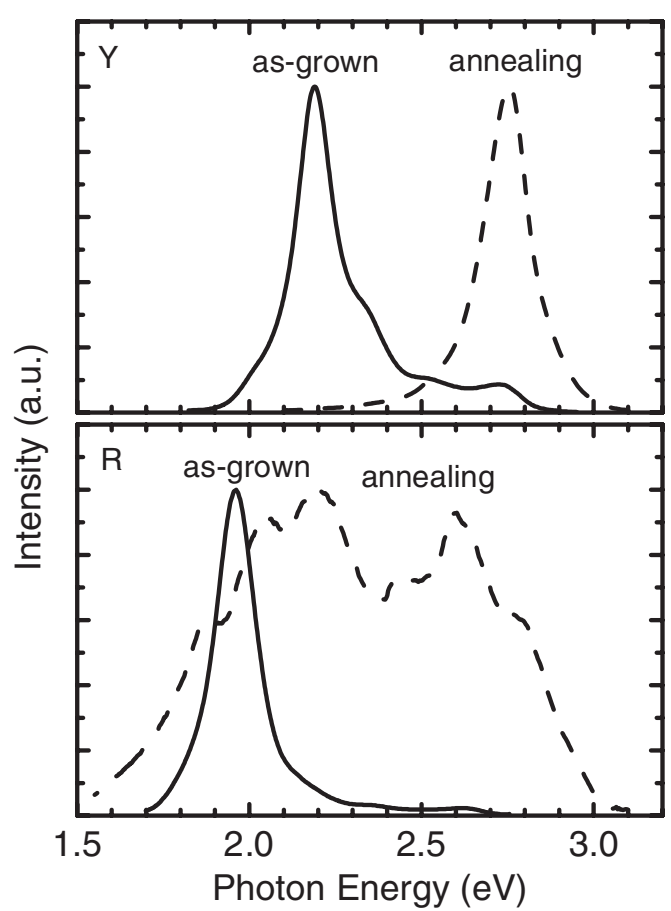

Fig. 1 PL spectra at $10 \mathrm{~K}$ before and after thermal annealing. a)

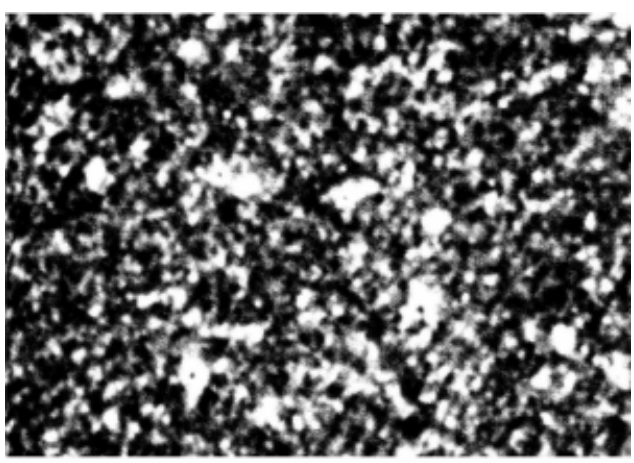

b)

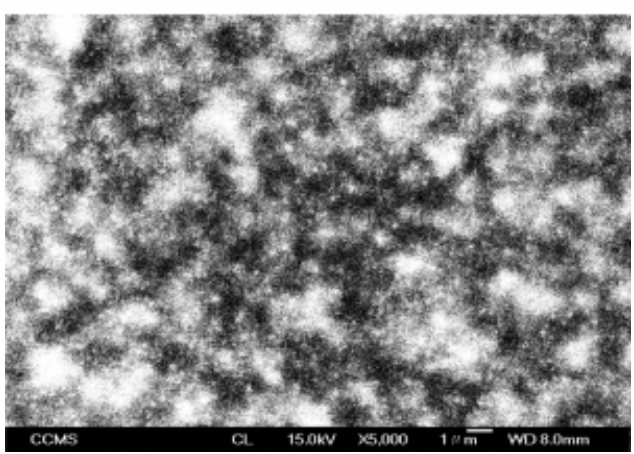

Fig. 2 Typical CL images of sample $\mathrm{Y}$ a) before and b) after thermal annealing. 
and relatively weaker blue light. After thermal annealing, the CL image (see Fig. 2b) becomes blurred because many tiny light spots of sub- $\mu \mathrm{m}$ in size were generated. Those tiny light spots essentially emit blue light. The generation of those tiny light spots implies that the cluster sizes in the InGaN film have been reduced upon thermal annealing, particularly in the shallower portion.

Figures $3 \mathrm{a}$ and $\mathrm{b}$ show the CL spectra of the as grown and annealed samples $\mathrm{Y}$, respectively. In each sample, the 3,5,8, and $15 \mathrm{kV}$ electron voltages roughly correspond to the penetration depths of 88,210 , 450 , and $1300 \mathrm{~nm}$, respectively. In the as-grown sample, mainly luminescence in the yellow band is observed in a shallow layer ( $3 \mathrm{kV}$ probe). With increasing electron voltage, not only the yellow band, but also the peak of blue luminescence (between 2.7 and $2.85 \mathrm{eV}$ ) can be observed. Also, with 8 and $15 \mathrm{kV}$ electron excitations, the luminescence from the GaN layer (around $3.4 \mathrm{eV}$ ) can be clearly observed. Hence, it is believed that a certain microstructure existing deep in the InGaN film can emit photons in the blue range. This result is consistent with the side-lobe of the PL spectrum in the as-grown sample, as shown in Fig. 1. After thermal annealing, in the shallow layer of InGaN ( $3 \mathrm{kV}$ probe), only blue luminescence exists that is again consistent with the PL measurement. With excitation of higher energy electrons, the blue luminescence is enhanced and the GaN emission peak appears. Also, a broad yellow luminescence is observed. This yellow luminescence is believed to originate from the defects in the $\mathrm{GaN}$ layer, instead of the cluster luminescence in the InGaN film. From the results described above, we speculate that after thermal annealing, the microstructure of the shallow layer of the InGaN film has been changed into that similar to the structure resulting in blue luminescence in the deeper portion. Hence, the whole InGaN film emits blue light. The different structures between the shallow and deep portions in the as-grown InGaN film is due to the fact that in growing the shallow portion, the high growth temperature has an effect of thermally annealing the deep portion.

Figure 4 shows the XRD patterns of the as-grown and annealed samples $\mathrm{Y}$. The InN peak (A) and the distribution (B), corresponding to InGaN of indium composition fluctuation, clearly confirm strong indium aggregation process in the as-grown and annealed samples. In the distribution $\mathrm{B}$, the signal split into two components after thermal annealing. The low-indium component is attached to the GaN peak. This phenomenon may imply a larger contrast of indium concentration between a cluster and the surrounding region. Hence, we can assume that during thermal annealing, spinodal decomposition tends to break large clusters into smaller ones. In this process, the indium content in an InGaN cluster can be enhanced. Meanwhile, the shrinkage of cluster size results in stronger quantum confinement and in-

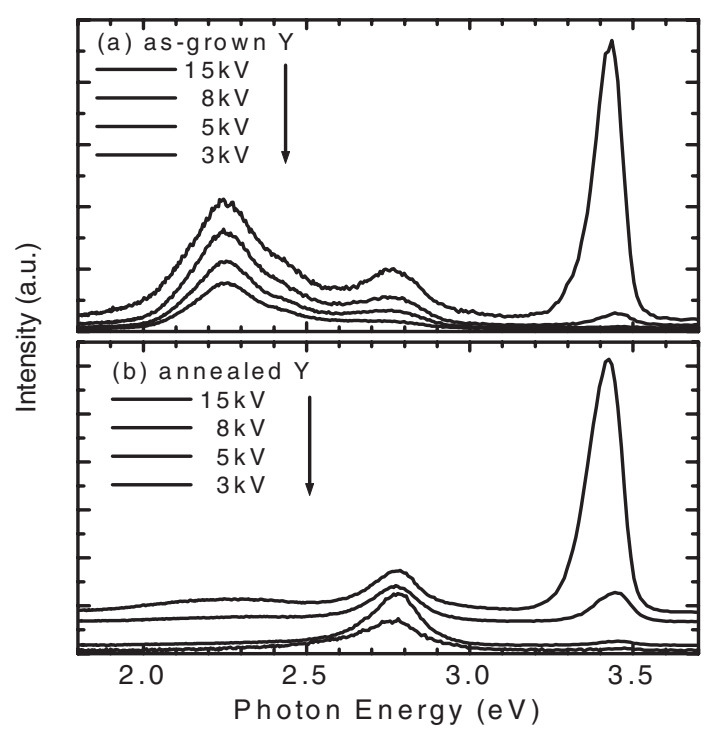

Fig. 3 Typical CL images of sample $\mathrm{Y}$ a) before and b) after thermal annealing.

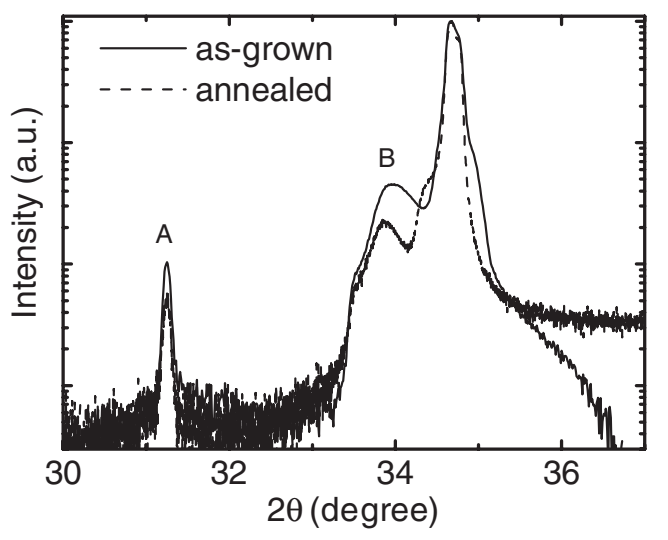

Fig. 4 XRD patterns of the sample Y before and after thermal annealing. 


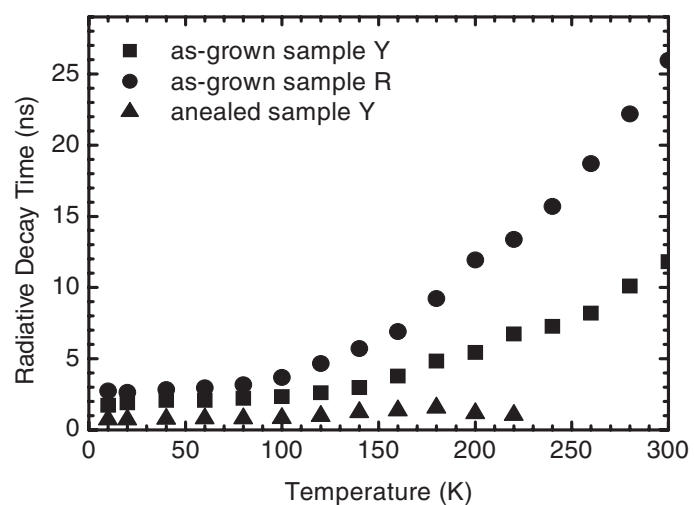

Fig. 5 Radiative decay times as functions of temperature at PL peak positions for as-grown samples $\mathrm{Y}$ and $\mathrm{R}$ and annealed sample $\mathrm{Y}$.

creases the effective band gap of the quantum dot, resulting in blue shift in PL spectrum. Furthermore, the strain within a cluster may be partly relaxed during the spinodal decomposition process upon thermal annealing. The reduction of QCSE can also contribute to the blue shift of PL peak.

To obtain the radiative decay time of excitions, we assume that the normalized integrated PL intensity versus temperature $T$ represents the internal quantum efficiency $\eta(T)$. We set $\eta(T)$ to be unity at $10 \mathrm{~K}$. The radiative decay time can be deduced from the PL decay time and cw PL integrated intensity [4]. Figure 5 shows the radiative decay times as functions of temperature at PL peak positions for as-grown samples $\mathrm{Y}$ and $\mathrm{R}$ and annealed sample $\mathrm{Y}$. The radiative decay time variations of as-grown samples $\mathrm{Y}$ and $\mathrm{R}$ show that the excitons behavior in some structures between 0-D and 3-D. The radiative decay time of the annealed sample $\mathrm{Y}$ is almost independent of temperature, suggesting that excitons behavior in quantum dot structures. This result is consistent with the assumption that during thermal annealing, spinodal decomposition tends to break large clusters into more quantum-dot-like InGaN clusters.

3 Conclusions In summary, we have studied the optical and material properties of two high-indium InGaN thin layers with luminescence in the yellow and red range and their post-growth thermal annealing effects. For one of them, the major part of PL spectrum was shifted from the yellow band into the blue range upon thermal annealing. CL spectra showed that the spectral shift occurred essentially in the photons emitted from the shallow layer of the InGaN film. The deeper layer in the as-grown film contributed blue emission because this portion has been thermally annealed during the growth of the shallow layer. The spectral shift was mainly attributed to the shrinkage of cluster size through spinodal decomposition upon thermal annealing. The attribution was supported by the observations in the CL, XRD, TRPL and HRTEM results.

Acknowledgements This research was supported by National Science Council, The Republic of China, under the grants of NSC 91-2215-E-002-030 and NSC 91-2215-E-002-034, and by US Air Force under the contract AOARD-02-4052.

\section{References}

[1] F. B. Naranjo, M. A. Sánchez-García, F. Calle, E. Calleja, B. Jenichen, and K. H. Ploog, Appl. Phys. Lett. 80, 231 (2002).

[2] E. F. Schubert, I. D. Goepfert, and J. M. Redwing, Appl. Phys. Lett. 71, 3224 (1997).

[3] T. Hino, S. Tomiya, T. Miyajima, K. Yanashima, S. Hashimoto, and M. Ikeda, Appl. Phys. Lett. 76, 3421 (2000).

[4] S. W. Feng, Y. C. Cheng, Y. Y. Chung, C. C. Yang, Y. S. Lin, C. Hsu, K. J. Ma, and J. I. Chyi, J. Appl. Phys. 92, 4441 (2002). 